\title{
Full field modeling of dynamic recrystallization in a global level set framework, application to $304 \mathrm{~L}$ stainless steel
}

\author{
Romain Boulais-Sinou ${ }^{1, a}$, Benjamin Scholtes ${ }^{1}$, Daniel Pino Muñoz ${ }^{1}$, Charbel Moussa ${ }^{1}$, Isabelle Poitrault ${ }^{2}$, Isabelle Bobin ${ }^{3}$, \\ Aurore Montouchet ${ }^{3}$, Marc Bernacki ${ }^{1}$ \\ ${ }^{1}$ MINES ParisTech, PSL Research University, CEMEF - Centre de mise en forme des matériaux, CNRS UMR 7635, CS 10207, rue Claude \\ Daunesse 06904 Sophia Antipolis Cedex, France \\ ${ }^{2}$ Industeel, ArcelorMittal, CRMC 71200 Le Creusot, France \\ ${ }^{3}$ Areva Creusot Forge, 6 allée J. Perrin 71200 Le Creusot, France
}

\begin{abstract}
A new full field numerical approach for the simulation of dynamic and post-dynamic recrystallization will be detailed. A level Set framework is employed to link a crystal plasticity finite element method with the modeling of recrystallization. Plasticity is calculated through the activation of slip systems and provides predictions for both SSDs and GNDs densities. These predictions control the activation and kinetics of recrystallization. All the developments are applied on 304L stainless steel.
\end{abstract}

Keywords: Recrystallization, CPFEM, Level Set.

\section{Introduction}

The optimization of industrial forming processes relies on the understanding of the material behaviour under the deformation conditions. The mechanical and thermal properties of metallic materials are inherited from their microstructure properties. Metals are formed of microscopic-length crystals, also called grains, which evolve when they undergo large plastic strain and high temperature. It is essential to control the microstructural evolutions with regard to the final in-use properties.

During hot working, the energy is mainly released through heat dissipation. However, a small part of the energy remains stored in the material under the form of crystallographic defects, principally dislocations. Many mechanisms can then be activated to minimize this energetic potential, such as recovery, grain growth (GG) or recrystallization $(\mathrm{ReX})$. The rearrangement of the microstructure during $\operatorname{ReX}$ is a complex phenomenon that is highly investigated in the literature [1]. During $\mathrm{ReX}$, dislocations-free grains, called nuclei, emerge from atomistic rearrangement and growth over the old deformed grains. The nucleation of new grains can occur during the deformation (dynamic recrystallization, DRX) and continue during an annealing treatment (postdynamic recrystallization, PDRX), or it can be fully activated after the dynamic step during this posttreatment (static recrystallization, SRX).

An increasing number of industrial companies show a strong interest in modeling the microstructure evolutions at small scale. In that context modeling means to understand/predict some physical phenomena at work in ReX mechanisms, such as the evolution of the stored energy, the strain hardening effect of plastic deformation or the prediction of the grain size and orientation distributions. This understanding can be obtained either through the development of simplified models (mean field approaches, MFA) or through the development of deterministic finite element (FE) methods where the microstructure topology is described (full field approaches, FFA). Both approaches need to be linked with experimental data to ensure that the models fit well with the real physical phenomena. The FFA can be divided into two main categories (probabilistic or deterministic). The probabilistic methods, such as the Monte-Carlo [2] or the Cellular Automata [3] approaches which are based on a stochastic evolution of the state variables on a voxel grid. The deterministic methods rely on solving systems of partial differential equations (PDEs). The later are more precise but also more time consuming. Due to this limitation, the PDE systems are solved on a Representative Volume Element (RVE). RVE's must be as small as possible while being big enough to contain all the heterogeneities of the microstructure. The Vertex method [4] is based on a front tracking description of the microstructure. This explicit description can encounter difficulties to handle topological events such as the appearance or disappearance of grains mainly in 3D. Thus front capturing approaches, like the Phase field method (PF) [5][6] and the level set method (LS) [6-9] seem more adapted to the simulation of ReX. The use of a FE

a Corresponding author: romain.boulais-sinou@mines-paristech.fr 
framework and the ad hoc meshing/remeshing capabilities allows also to reach large deformations of RVEs, which is not straightforward when dealing with regular grids such as in the Fast Fourier Transform methods (FFT) [10]. The PF formulation requires additional numerical parameter, like the interface width, whereas the LS formulation only relies on measurable physical and measurable quantities, which makes it more direct to use for solving the PDE systems of Crystal Plasticity Finite Element Method (CPFEM) and DRX.

This paper presents a full field approach in a LS framework and the first steps of its application to the simulation of DRX in 304L stainless steel.

\section{Modelling of dynamic recrystallization}

In this section, the solving of crystal plasticity problem using the FE method is presented. First we introduce the governing equations and the crystal plasticity PDE systems. Then, we describe the LS formulation used to represent the polycrystal RVE and the grain boundary motion due to the stored energy.

\subsection{Crystal Plasticity Finite Element Method}

The behaviour of the polycrystal during the deformation is described by a CPFEM model, based on a classical elasto-viscoplastic formulation in a Lagrangian formalism. We use the classic decomposition of the deformation gradient into an elastic and a plastic part.

$$
F=F_{e} F_{p}
$$

In crystalline matter, the accommodation of plastic deformation $F_{p}$ is done through the activation of slip systems at the atomistic level. When the local stress on an atomic plan reaches a critical value, the atoms start to glide to accommodate the macroscopic deformation and create atomistic defects, mainly dislocations. In FCC material, there are twelve crystallographic slip systems $\{111\}<110>$ that are likely to be activated with the plastic deformation. For each slip system $\alpha$, we need to determine the average velocity of a dislocation gliding. Here, the slip rate $\dot{\gamma}_{\alpha}$ is calculated from the following viscoplastic exponential law:

$$
\dot{\gamma}_{\alpha}=\dot{\gamma}_{0}\left|\frac{\tau^{\alpha}}{\tau_{c}^{\alpha}}\right|^{1 / m} \operatorname{sign}\left(\tau^{\alpha}\right)
$$

where $\dot{\gamma}_{0}$ is the reference slip rate, $m$ is the sensitivity exponent, $\tau^{\alpha}$ is the local shear stress on slip system and $\tau_{c}^{\alpha}$ is the critical resolved shear stress (CRSS) required to activate the gliding. $\tau^{\alpha}$ is related to the macroscopic stress $\bar{\sigma}$ through the Schmidt tensor $\overline{\bar{T}}^{\alpha}$, which is calculated for each slip system $\alpha$,

$$
\tau^{\alpha}=\overline{\bar{T}}^{\alpha}: \bar{\sigma}
$$

For a FFC material, $\tau_{c}^{\alpha}$ is supposed to be the same for all the slip systems, noted $\tau_{c}$, and it is obtained solving the following dislocation-based hardening law:

$$
\tau_{c}=\tau_{0}+\psi \mu b \sqrt{\rho}
$$

with $\tau_{0}$ the initial CRSS, $b$ the Burger's vector norm, $\mu$ the shear modulus and $\psi$ a hardening coefficient to be identified with experimental data and chosen in the following as $1 / 2$. The prediction of the dislocation density $\rho$ is then of prime importance for the integration of the behavior law. In this work, two type of dislocation densities are considered and the total dislocation density is the sum of those two dislocation populations. The statistically stored dislocation (SSD) is related to the velocity gradient $V_{p}$ with the Yoshie-Laasraoui-Jonas formulation [11]:

$$
\left\{\begin{array}{c}
\dot{\rho}_{S S D}=\left(\frac{K_{1}}{M}-\frac{K_{2}}{M} \rho_{\text {total }}\right) V_{p}, \\
V_{p}=\sum_{\alpha}\left|\dot{\gamma}_{\alpha}\right| \\
\rho_{\text {total }}=\rho_{S S D}+\rho_{G N D}
\end{array}\right.
$$

where $K_{1}$ and $K_{2}$ are material parameters related to the storage and annihilation of dislocations, respectively, and $M$ is the Taylor factor. The geometrically necessary dislocations (GND) accumulate in regions of plastic gradient such as the grain boundaries in order to satisfy the geometric continuity. Hence they are predicted from the gradient of plastic deformation $\mathbf{F}_{p}$ presented in [12]:

$$
\left\{\begin{array}{c}
\dot{\boldsymbol{\rho}}_{G N D_{\alpha}}=\frac{1}{b} \nabla \times\left(\dot{\gamma}_{\alpha} \boldsymbol{n}_{\alpha} \mathbf{F}_{p}\right), \\
\dot{\rho}_{G N D}=\sum_{\alpha}\left\|\dot{\boldsymbol{\rho}}_{G N D_{\alpha}}\right\|_{L_{1}},
\end{array}\right.
$$

with $\boldsymbol{n}_{\alpha}$ the slip direction of the $\alpha$ system. In the end, the PDE system can be summarized as shown in figure 1 .

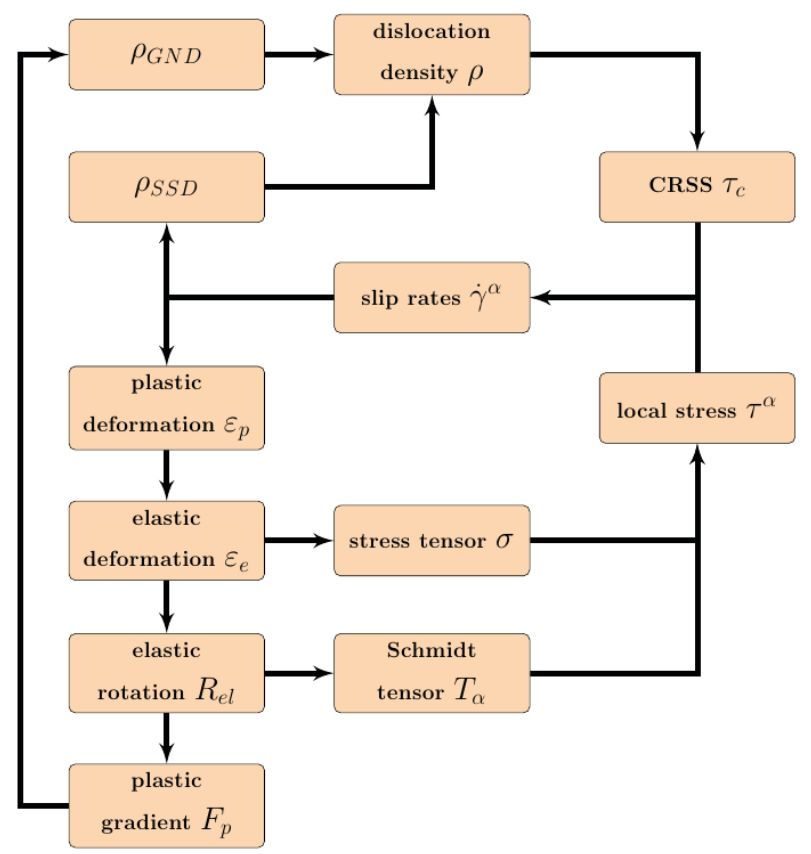

Figure 1. Flow chart showing the coupling between the crystal plasticity state variables.

\subsection{Level-Set formulation}

The LS method is used to describe the interfaces, here the grain boundaries. At each interpolation point of the finite element mesh (P1 interpolation), the LS function $\psi$ 
corresponds to the signed distance to the interface $\Gamma$ of a sub-domain $\Omega$. The position of an interface is known though the isovalue $\psi=0$ of its corresponding LS function. The sign convention used here is $\psi \geq 0$ inside $\Omega$ and $\psi \leq 0$ outside $\Omega$.

$$
\left\{\begin{array}{l}
\psi(x, t)= \pm d(x, \Gamma(t)), x \in \Omega \\
\Gamma(t)=\{\psi(x, t)=0, x \in \Omega\} .
\end{array}\right.
$$

In the case of ReX, the displacement of the LS interface is done in an Eulerian way by solving the following transport equation according to a given velocity field $\vec{v}$.

$$
\left\{\begin{array}{l}
\frac{\partial \psi(x, t)}{\partial t}+\vec{v} \cdot \nabla \psi(x, t), \\
\psi(x, t=0)=\psi_{0}(x) .
\end{array}\right.
$$

For a grain boundary, the velocity $\vec{v}$ is a combination of the stored energy potential between the neighboring grains and the capillarity force at the interface,

$$
\vec{v}=\vec{v}_{\Delta \text { Energy }}+\vec{v}_{\text {capillarity }}
$$

The energy gradient between two neighboring grains $\Delta E_{n e r g y}=E_{\text {grain }_{i}}-E_{\text {grain }_{j}}$, is calculated from the average dislocation densities of the grain $i$ and $j$.

$$
E_{i}=\int_{x \in \operatorname{grai} \square i}\left[\frac{1}{2} \mu b^{2} \rho(x)\right] d x .
$$

When working with large polycrystal, the number of LS functions required to describe all the grains can be very high. In this paper, we work with the Global Level Set (GLS) framework developed by Scholtes et al. and described in [13] for GG and [14] for SRX. The GLS methodology enables us to describe more than one grain with one LS function. The full fields and mean fields inherent to each grain are conserved individually and the problematics due to topological events, like grain coalescence, are handled by swapping the grains from one GLS to another. In the following, GGReX will denote the algorithm developed in [7][8] and improved in $[13][14]$ to model grain boundary motion due the capillarity force and the stored energy.

\subsection{Grain Growth and Recrystallization model}

If we consider the $\operatorname{ReX}$ phenomenon modeling, we observe that the overall stored energy of the system must decrease. Physically, when a grain boundary is moving, it leaves a dislocation-free area behind. To reproduce this phenomenon in our FFA model, we chose to reset the dislocation densities SSD and GND behind the recrystallization front. Thanks to the LS functions describing the grain boundaries, it is easy to identify which area has been recrystallized and reset the energy field at the corresponding mesh elements. Figure 2. illustrates how elements are identified behind the front (red hatched elements). At the atomistic level, the rearrangement of atoms behind the recrystallization front changes the local lattice orientation. It is commonly accepted that the new orientation in this area will be similar to the orientation of the grain it has grown from. To deal with this local orientation change, we chose to extend the orientation field of the growing grain behind the recrystallized front as shown in Figure 3. Capillarity effects (GG) are also taken into account on the proposed formalism as described in [8][13][14].

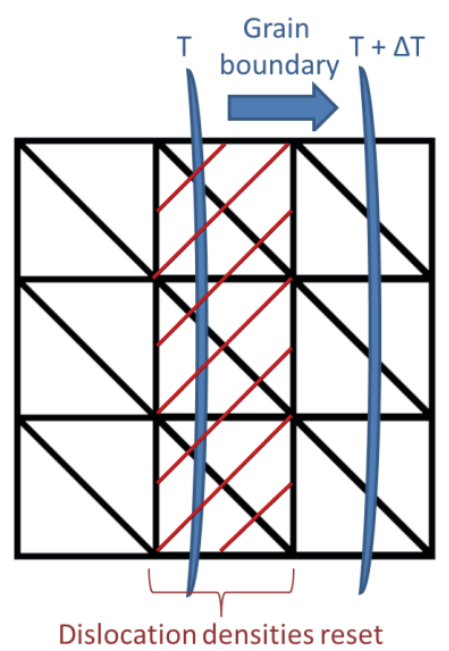

Figure 2. Identification of the recrystallized element and resetting of the dislocation density.

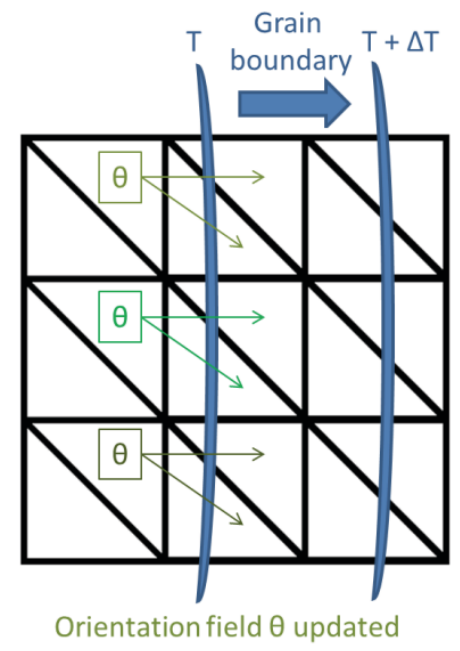

Figure 3. Extension of the orientation field from the growing grain onto the recrystallized elements.

\subsection{Nucleation model}

The nucleation events occur when the dislocation density reaches a critical value, meaning that the material has stored more energy than it can handle. In previous work at CEMEF [15][16], criteria for both SRX and DRX nucleation were proposed. For the DRX model, the critical dislocation density $\rho_{c r}$ is obtained as a limit from a sequential equation:

$$
\left\{\begin{array}{c}
\rho_{c r_{0}}=\left(\frac{20 K_{1} \gamma \dot{\varepsilon}}{3 M \tau^{2}}\right)^{\frac{1}{3}}, \\
\rho_{c r_{n+1}}=\left[\frac{-2 \gamma \dot{\varepsilon} \frac{K_{2}}{M \tau^{2}}}{\ln \left(1-\frac{K_{1}}{\theta K_{2}} \rho_{c r_{n}}\right)}\right]^{2},
\end{array}\right.
$$


with $\dot{\varepsilon}$ the macroscopic strain rate, $\gamma$ the interfacial energy, $M$ the mobility of the interface, $\theta$ a constant whose value is close to 0.3 [15] and $\tau$ the dislocation line energy. When an element of the domain reaches the critical value $\rho_{c r}$, a spherical new grain is introduced. In order to ensure that the nucleus do not shrink as soon as it appears, its initial radius $R_{i n i}$ is chosen greater than the local critical radius $R_{c r}$ defined as follow:

$$
R_{i n i}>R_{c r}=\frac{2 \gamma}{\rho_{c r} \tau}
$$

All nuclei formed are dislocation-free new crystals. The initial dislocation density of a nucleus is then set equal to an annealing configuration, $\rho_{i n i}=10^{11} \mathrm{~m}^{-2}$. At their creation, we assume that their orientation $\theta$ is the same as the region of the parent grain they grow from. To model this aspect, the initial orientation field inside is set to the value $\theta_{0}$ from the center element of the nucleus, i.e. the element chosen to introduce the nucleus. Figure 4 illustrates how a new grain is introduced on the FE mesh.

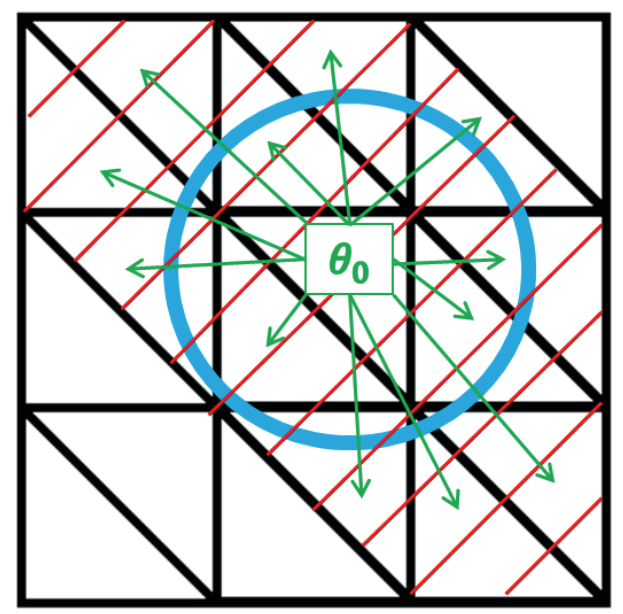

Figure 4. Introduction of a new germ (blue LS). Dislocation density and orientation field are reset on the red hatched element.

\section{Case Studied}

In order to test our formulation we built a classical Channel-Die test where a 3D polycrystal RVE is deformed. The material investigated here is $304 \mathrm{~L}$ stainless steel. All experimental data were obtained from hot torsion tests under constant strain rate $\dot{\varepsilon}=10^{-2} \mathrm{~s}^{-1}$ and constant temperature $T=1000^{\circ} \mathrm{C}$ [14-16]. However, for the first simulations described in the following, we fixed a lower strain rate value equal to $\dot{\varepsilon}=2.78$. $10^{-6} s^{-1}$ that corresponds to $1 \%$ strain over one hour. The objective is to accurately observe the evolution of grain boundaries, orientation field and dislocation densities while the deformation on the RVE does not exceed $3 \%$. The initial RVE is a $1 \times 1 \times 1 \mathrm{~mm}^{3}$ cubic polycrystal that contains 200 grains for a mean grain size $\bar{D}=212 \mu \mathrm{m}$. To generate the microstructure, a
Voronoï tessellation and a LS immersion are used [17]. Boundary conditions are imposed on all RVE external faces and respect the constant strain rate $\dot{\varepsilon}$ described previously. The mesh used for this simulation contained roughly 100000 elements. Also, an adaptive mesh refinement [18] is performed every 5\% strain level. At this validation stage, the impact of the chosen mesh size was not yet investigated. In order to represent an initial annealed state for the material, the initial GND density is null $\rho_{G N D_{0}}=0 \mathrm{~m}^{-2}$ and the SSD density is set at an estimated value of $\rho_{S S D_{0}}=10^{11} \mathrm{~m}^{-2}$. The CPFEM and GGReX models are coupled following the global algorithm detailed in the flow chart in Figure 5. The global time step is fixed and adapted during the simulation as the minimum of the time step values required by the CPFEM and GGReX models.

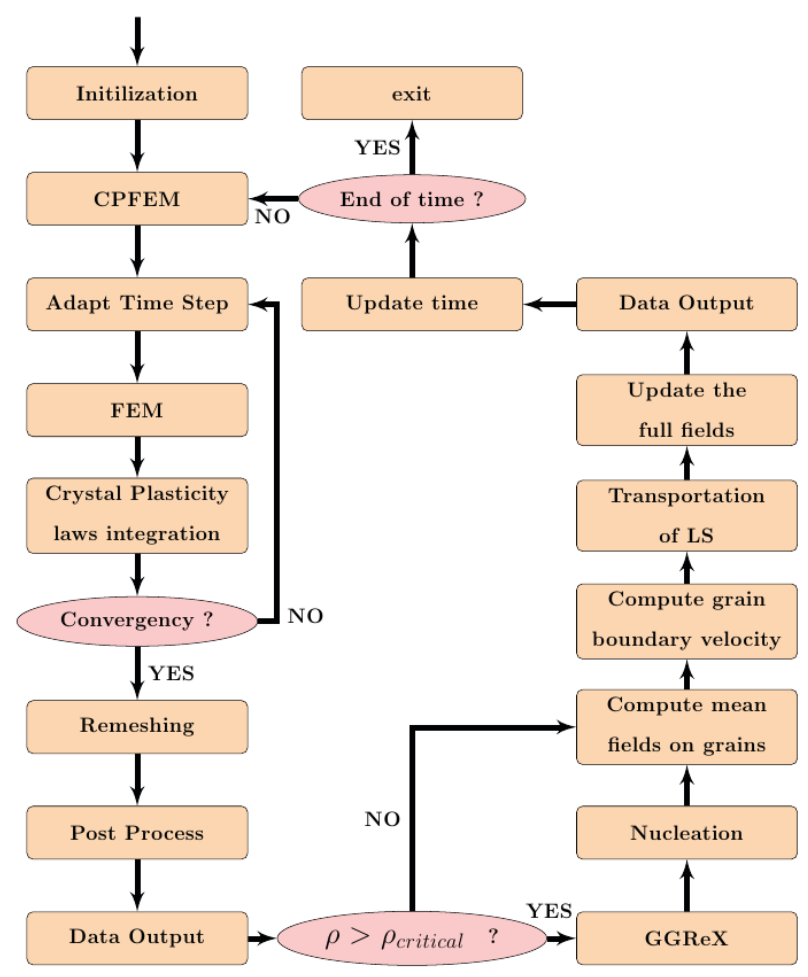

Figure 5. Coupling between CPFEM and GGReX.

\section{Primary results}

In the early stage of the simulation, only CPFEM is at work and the grains are accumulating energy. When the energy gradient between grains becomes significant it activates the GG/ReX mechanisms. Under the constant strain rate and temperature condition imposed from experimental data, we observe that the energy reduction through the grain boundary migration is too important to reach the experimental critical value calculated at $\rho_{c r}=$ $1.8 \cdot 10^{13} \mathrm{~m}^{-2}$ from Eq. (11). Hence there is no nucleation step in these results and a Strain Induced Boundary Migration (SIBM) configuration is observed. Fig. 6 illustrates the decrease of the dislocation density due to the effect of the grain boundary migration (SIBM). Fig. 7 and Fig. 8 illustrate, respectively, the evolution of 
the first component of the orientation field and the evolution of the dislocation density field (in $\mathrm{m}^{-2}$ ).

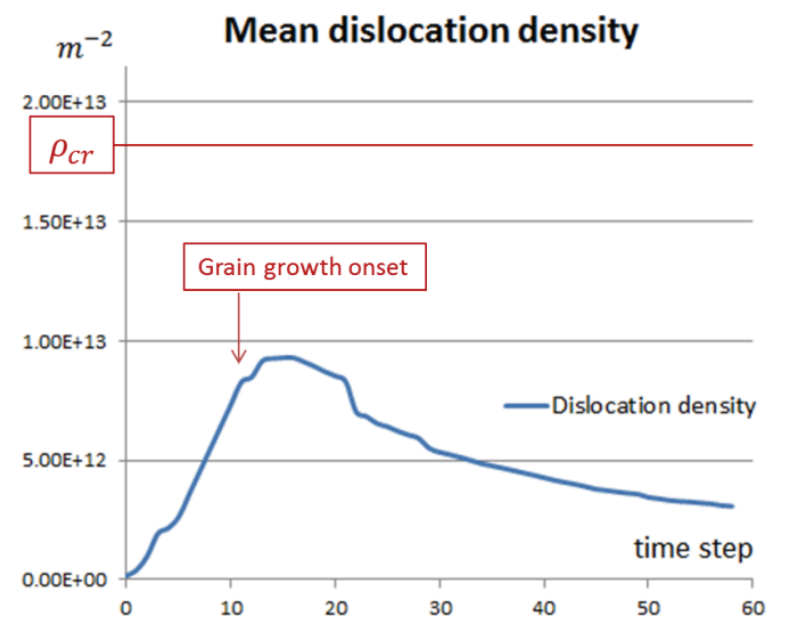

Figure 6. Evolution of the mean dislocation density in the RVE.

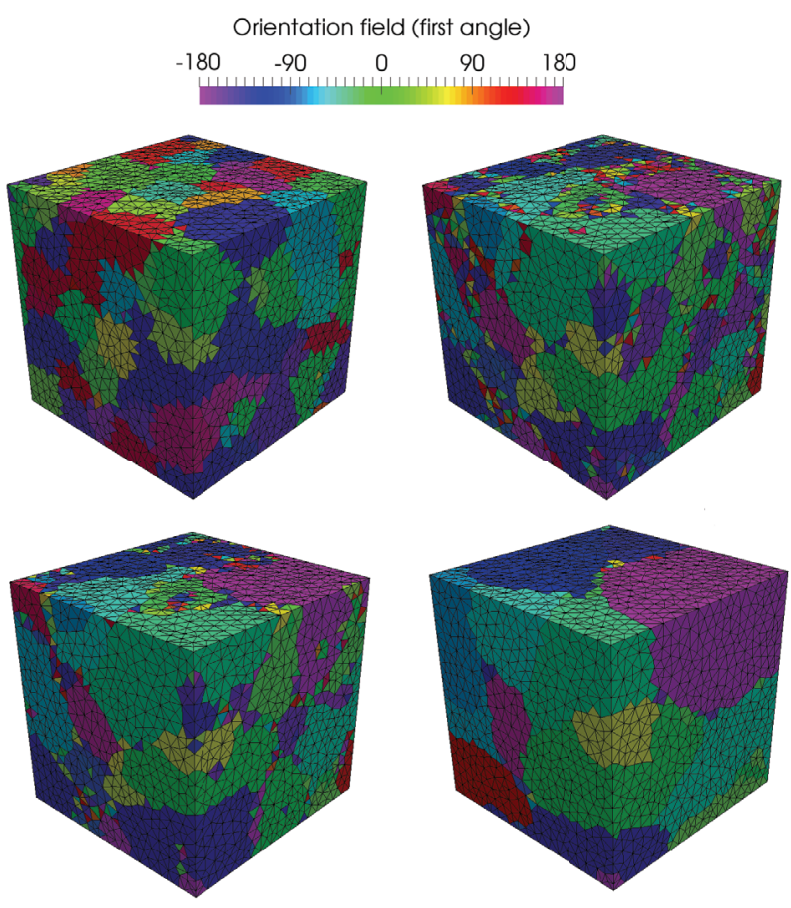

Figure 7. First component of the orientation field $\in$ $\left[-180^{\circ}, 180^{\circ}\right]$. From left to right and top to bottom: initial field and fields after 150, 300 and 600 time steps.

\section{Perspectives}

This coarse case is a first step toward the simulation of DRX. Further testing of the described coupling between the crystal plasticity model and the GGReX model for finer FE mesh must be carried out. First, we will perform simulations using the same thermomechanical conditions as the experimental torsion tests. Our numerical DRX model will then be validated thanks to experimental data concerning 304L steel material.

Then, it will be interesting to observe the RVE behavior with the introduction of the dislocation-free grains. Once the nucleation happened, the softening effect on the strain stress response will be investigated. For instance, we could observe the effects of higher strain rate conditions or lower temperatures. The orientation dependence of the nuclei could also be an interesting topic research. Complex industrial pass leading to DRX phenomena will be investigated. Finally, these developments will be implemented in the DIGIMU $®$ software package [19].

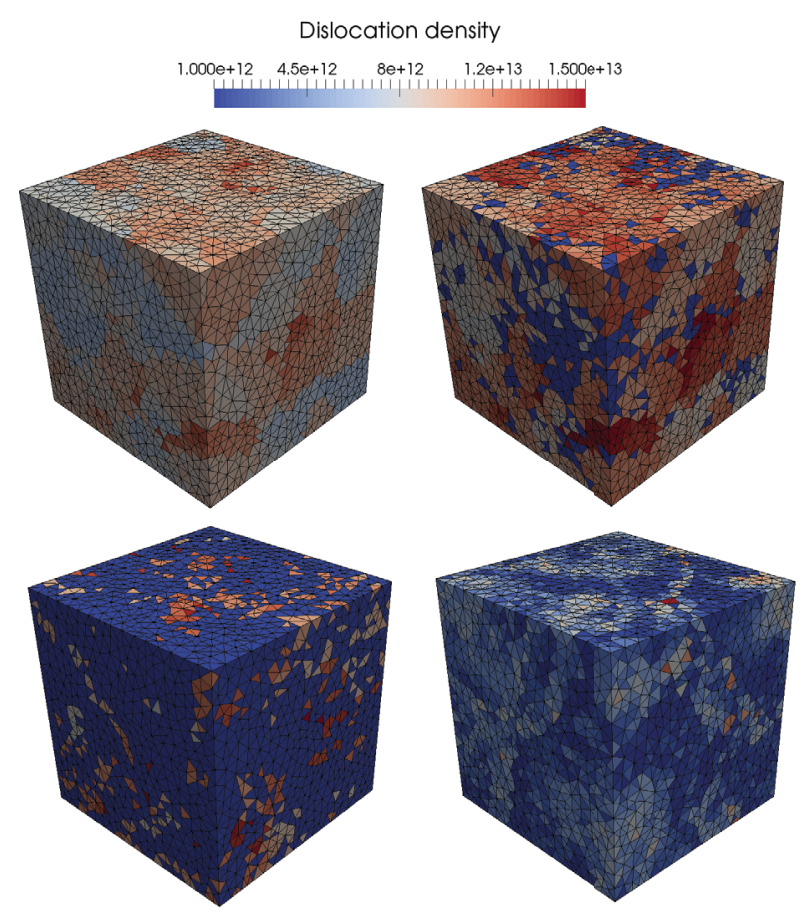

Figure 8. Evolution of the dislocation density field $\rho$ $\left(m^{-2}\right)$. From left to right and top to bottom: initial field and fields after 150, 300 and 600 time steps.

\section{Acknowledgements}

The authors would like to gratefully thank ArcelorMittal Industeel, Areva, Ascometal, Aubert \& Duval, CEA and Safran for funding this research through the MICROPRO2 consortium.

\section{References}

1. A. D. Rollett, Overview of modeling and simulation of recrystallization, Progress in Materials Science, 42, 79 99 (1997).

2. K. Okuda and A.D. Rollett, Monte Carlo simulation of elongated recrystallized grains in steels, Computational Materials Science, 34, 264 - 273 (2005).

3. G. Kugler and R. Turk, Modeling the dynamic recrystallization under multi-stage hot deformation, Acta Materialia, 52, 4659 - 4668 (2004).

4. K. Piekos, J. Tarasiuk, K. Wierzbanowski, B. Bacroix, Generalized vertex model of recrystallization Application to polycrystalline copper, Computational Materials Science 42, 584-594 (2008).

5. R. Darvishi Kamachali, S. Kim, I. Steinbach, Texture evolution in deformed AZ31 magnesium sheets: Experiments and phase-field study, Computational Materials Science 104, 193-199 (2015). 
6. Y. Jin, N. Bozzolo, A.D. Rollett, and M. Bernacki, 2D finite element modeling of anisotropic grain growth in polycrystalline materials: level set versus multi-phase-field method, Computational Materials Science, 104, 108-123 (2015).

7. M. Bernacki, H. Resk, T. Coupez, and R. Logé, Finite element model of primary recrystallization in polycrystalline aggregates using a level set framework, Modelling and Simul. Mater. Sci. Eng. 17, 064006 (2009).

8. M. Bernacki, R. Logé, and T. Coupez, Level set framework for the finite-element modelling of recrystallization and grain growth in polycrystalline materials, Scripta Materialia, 64, 525-528 (2011).

9. M. Shakoor, B. Scholtes, P.-O. Bouchard and M. Bernacki, An efficient and parallel level set reinitialization method Application to micromechanics and microstructural evolutions, Applied Mathematical Modelling, 39, 7291 7302 (2015).

10. B. Liu, D. Raabe, F. Roters, P. Eisenlohr and R.A. Lebensohn, Comparison of finite element and fast fourier transform crystal plasticity solvers for texture prediction, Modelling and Simul. Mater. Sci. Eng. 18, 085005 (2010).

11. A. Laasraoui and J.J. Jonas, Prediction of steel flow stresses at high temperatures and strain rates, Metallurgical Transactions A, 22, 1545-1558 (1991).

12. E.P. Busso, F.T. Meissonnier and N.P. O'Dowd, Gradientdependent deformation of two-phase single crystals, Mechanics and Physics of Solids, 48, 2333-2361 (2000).

13. B. Scholtes, M. Shakoor, A. Settefrati, P.-O. Bouchard, N. Bozzolo and M. Bernacki, New finite element developments for the full field modeling of microstructural evolutions using the level-set method, Computational Materials Science, 109, 388-398 (2015).

14. B. Scholtes, R. Boulais-Sinou, A. Settefrati, D. Pino Muñoz, I. Poitrault, A. Montouchet, N. Bozzolo, and M. Bernacki, 3D level set modeling of static recrystallization considering stored energy fields, Computational Materials Science, 122, 57-71 (2016).

15. K. Huang, Towards the modelling of recrystallization phenomena in multi-pass conditions: application to 304L steel, PhD thesis, MINES ParisTech (2011).

16. A. L. C. Fabiano, Modelling of crystal plasticity and grain boundary motion of 304L steel at the mesoscopic scale, $\mathrm{PhD}$ thesis, MINES ParisTech (2012).

17. K. Hitti, P. Laure, T. Coupez, L. Silva and M. Bernacki, Precise generation of complex statistical Representative Volume Elements (RVEs) in a finite element context, Computational Materials Science, 61, 224 - 238 (2012).

18. H. Resk, L. Delannay, M. Bernacki, T. Coupez and R. Logé, Adaptive mesh refinement and automatic remeshing in crystal plasticity finite element simulations, Modelling and Simul. Mater. Sci. Eng. 17, 075012 (2009).

19. B. Scholtes, M. Shakoor, N. Bozzolo, P.-O. Bouchard, A. Settefrati, and M. Bernacki, Advances in level-set modeling of recrystallization at the polycrystal scale development of the digi- $\mu$ software, Key Engineering Materials, 651-653, 617-623 (2015). 